\title{
Study on Urban Ecological Landscape Design Methodology Based on the Interaction Philosophy
}

\author{
Dong CHU \\ Department of Environmental Art and Design \\ Harbin University of Commerce \\ Harbin, China \\ e-mail: chudongcd2007@sina.com
}

\begin{abstract}
The passive linear-thinking of the industrial society has been replaced by the interactive cross-thinking, resulted in the interaction design which is a new design method to observing the world. This design method is a synthesis of dynamic, virtualization, experience and emphasizes the intersectionality, integrity and scientificity. The thesis focus on the ideological connotation and the according design method taking landscape as a media, which expects to intervene the urban landscape design practice. Meanwhile, the interdisciplinary research is also taken into consideration with the conception integration to achieve the scientific design about the behavior experience in the process of the landscape interaction design. The goal is to solve the practical problems of urbanization in China with the landscape design intervention.
\end{abstract}

Keywords-landscape design; interaction design; dynamic; virtualization; experience

\section{THE EXISTING CIRCUMSTANCE OF URBAN LANDSCAPE DESIGN}

In the process of the urbanization in China, rapid removal and reconstruction lead to the absence of the comprehensive plan and ecological conception. The topographic and geological features, water, vegetation are always neglected. Some of the projects are applying mechanically and destroyed the intrinsic natural relationship of the area. Due to the lack of the consideration on the design connotation, blindly copy and unprincipled collage, some of the cities in China are suffering from uniformity and they are facing similar problems. The European and the Roman styles of landscape are arising like the mushrooms after the rain. The hard top, river diversion and exotic tree species are applying everywhere, which caused the ecological imbalance. This linear thinking neglects the secondary relationship between the physical forms and its inner core, the interaction relationship between human and the nature. The passive design method focusing on the forms can not bring the spirit texture of human in line with the texture of the nature. This is the main reason for the design forms deviating from our daily life and never plays its real role as the urban landscape.

\section{WHAT IS INTERACTION DESIGN}

Every important invention doesn't achieved by its own discipline, but the theory coming from other subjects.
Modern design has gone through the alternative changes between the form and the connotation. In the information age, human behaviors are being concerned, which means the interaction between complex system and human is taking into consideration. In brief, interaction design is about the behaviors of the artifacts, environment and its system connections, including the form design for it. It is different from the traditional design which mainly focuses on the forms, but pay much attention on the content and purpose. Interaction design firstly aims at the planning and describing the manner of the behavior and then design the most effective form to express the behavior. As a subject of the interactive experience, interaction design was proposed by Bill Moggridge, founder of the IDEO in a meeting in 1984. Gillian Crampton Smith later applied this theory into design education and set up the Computer Related Design Department in RCA. Now many research center set up similar department in order to pursue the quantitative and qualitative investigation from definition, methodology and design criteria of different angles in the form of theory and practice.

\section{TRANSFORM FROM THE INTERACTION DESIGN THEORY TO THE DESIGN METHODOLOGY}

Design used to be imposed on some unnecessary principles. What is important in the context of the fast changing technology, designers should master the innovation of the design methodology, which derives the diversified development trend of design, but not only to copy the form. Actually, design is not a verb, but it is a practice process probing for the method. Design methodology is not the best way to solve the problem, but the pursuit for the scientific design method is the future development trend for the landscape design. The design method deriving from the interaction design is an integration of several characteristics, for example, the dynamic association, virtualization, experience, focusing on the integrity, intersection, scientific nature. It has got rid of the depending on the individual's subjectivity and emphasizing on the human behavior process design based on the scientific method and tools to analyze the overall changes. Design has changed from the old static analysis to the dynamic simulation in the computer, which promote design into a new age.

With the maturity of the landscape ecology theory, the theory of interaction design is applied widely into modern 
landscape design projects, emphasizing on scientific analysis about the every design steps in order to build a media between human and nature and create a comfortable living environment. Risser ET AL believes that landscape ecology is not an independent principle or works as a simple branch of the ecology, but an intrinsic synthesis of many relevant design philosophies, which focuses on the space-time form of the landscape. What the landscape ecology works on is about the relationship of the space functions in the ecosystem, including the integration between the structure and function of human activities in the ecosystem.

The theory firstly comes from the book Design with Nature written by Ian McHarg, which expresses the idea of the layers overlay. It is the key to generate the interactive relation by obtaining, analyzing and disposing all the data about the environment, transportation, economy, education, resources, and culture and so on. The landscape design based on the interactive design theory draws lessons from the engineering theory and technology. It is a synthesis of the new design methods, but not the partial stack. It aims at redesigning the usage mode by the method of the goal orientation, so as to redefine the relationship between the environment and human behavior, probing for the dialogue between culture and history. The interactive landscape design method could also forecast the extent of the impact between the human and the environment, focusing on human behavior design, which is the influence of landscape design on our life style.

\section{A. Dynamic Association}

The traditional design method always focuses on solving the single question itself, but interactive design put more attention on the connections of the problems. Design is becoming a dynamic process and changing into an interaction between the subject and the object, rather than the form of the landscape. James J. Gibson put forward the conception of "Affordance" in the book The Ecological Approach to Visual Perception, meaning that the property afforded by the environment for the animals.

Gibson explained Affordance by an example that if we take the land surface as the basement, we could judge the type of the activity and the people who take part in by the horizontality, rigidity, smoothness, extension of the land. All these four physical property could be measured only after they are associated with specific activities. And then the Affordance of environment becomes a two way interactive measurement rather than a fixed physical variable. Affordance contains not only physical factors but also psychological ones. It served for both the environment and people in the same time, shaping a resonance between them. Donald Norman took the concept of Affordance much further. He pointed that it means the environment could make people feel that they should do something. A good interactive design would guide people to take part in the activities on their own initiative. For example, a button is the thing that makes people want to press. And this is the Affordance. It works as a property, giving a hint and guidance for the public. Like the handle of a cup gives people the hint to hold as soon as they see it.
We have to create this Affordance actively in the process of the landscape design by building different kind of substance and space construction, which could afford people with the possibilities to communicate with each other and achieve the interactive connection between human and nature. This is not a form design, which is imposed on nature and people. "There is no need to give people an instruction to tell them how to use; it has to be intuitive which will guide people to use it consciously." Ian McHarg believes that earth has its intrinsic value. Land is alive, who is a living system. It tells us what we should do on the earth and what is the harmonious relationship between human and land. The way of thinking is to integrate all you ecological cognition together, which is called the method of thousand layers cake. The bottom layer is the geology, and then topography, vegetation, water, human activities. After a systematic suitability analysis about the land, designers could get a scientific idea about how to make good use of the land and how to protect it. Accordingly, landscape design is not a form design, but a connection design between human and nature based on this Affordance concept. It creates an experience process. Professor Kongjian Yu also concluded that if some of the basic factors are taking into account in advance, for example the social, economic, material elements, and then a better form and space will solve all the problems. Then everything is logical and correct.

\section{B. Interactive Virtualization}

Multidisciplinary research has laid a more solid theoretical foundation for the interactive design in the digital age. Multimedia has provided a greater diversity. The process of the interactive design contains input and output. People input instructions into the computer and the result comes out after a delicate processing. The form of this processing could be varied, just like the interaction between human and nature could derive a kind of landscape behavior. Roger Tomlinson set up Geographic Information System in 1966. The spacial analysis coming from the GIS is based on the data of the geographic location and the morphological character of the space. With the help of GIS, landscape designer could draw the traffic flow chart, economic distribution map, land use map, road grade map quickly. It could also overlap all these layers together and give you a comprehensive view just agree with theory of the layer overlay technology put forward by Ian McHarg.

GIS provides a better research method for landscape design. Landscape design has transformed from the traditional, single, direct thinking to the cross-disciplinary and comprehensive thinking. This thinking way could make up for the deficiencies of the two-dimensional landscape thinking and the lateral thinking of the software. GIS has set up vertical environment factors to improve the scientific and ecological characteristics of the whole design.

The traditional landscape design method creates a static visual experience with the software of Auto Cad, Photoshop and 3dmax. This old design conception set up on the perceptual experience. Modern landscape design research based on the interactive design analyzes the planned space data in view of the topographic map and remote sensing 
image making by GIS as the basic data sources. After setting up the DEM and overlapping the remote images of the threedimensional space, designers could get a good understanding about the local topography, spatial pattern and perspective. Finally, combined with the history culture of the local area, the interactive landscape design is built on the basis of the previous scientific analysis. Meanwhile, GIS could achieve a dynamic interaction among human, software and environment after a virtual simulation analysis. It will provide dynamic geographic space information timely and realize the scientific design of the behavior experience in the process of the modern landscape interactive design, which build an interactive connection between human and nature.

\section{Emotional Experience}

"Good design not only meets the function, but also inspires positive emotion of the users. It will also build a emotion connection between the designers and the users." Design is an interactive product which support human's everyday life. Interactive design focus on the target users expectations and the behavior in the process of the interaction. An effective interaction can be got after portraying human behavior and psychological model. The application of the interactive design concept into landscape design is a behavior experience process design based on landscape as the media. It is the most important experience between human and environment. You can experience it with your body and feel it with your heart. It is the emotion and consciousness coming from human activities. This is a process in which the subject takes part in the activity and sublimates its emotion after interacting with the object. We are living in the experience economy era in which people are pursuing to meet the spiritual and psychological needs. Alvin Toffler said that arts in this era produce a different kind of experience because of their own purpose. Actually, environmental artists are becoming the engineers who produce experimental arts.

People are looking forward to combine living space with nature and obtaining spiritual and emotional interactive experience from the urban landscape design, but not to bend to the visual appearance. The form of the landscape design will be better because of the emotion and the emotion will appear in the form. Landscape design is a life connection between human and nature. It is a living experience process. Space is no longer taken as a physical factor in the concept of the post-modernism in the post-industry era, but an experience. Space should be perceived, emphasizing the relationship with human experience and emotion. The emotional process returns to the nature of the object, and then achieves the interactive process between the spirit of the subject and the form of the object.

\section{The Idea of Integration}

"Urban design is performing the function of arranging the space, time, meanings and contact in harmony. The organization and meanings of the spaces are the intrinsic qualities of urban design which could be interpreted into the tangible expression of people's ideal environment." The image of the urban landscape is a kind of space design, which is three-dimensional. We should attach importance to the spatial relations, if we focus on the aesthetic visual ecology. For example, the form, size, density, capacity, connections and sequence. The adverse impact coming from the harmful eyesight should be reduced. The artificial environment should appear naturally with its visual diversity. Meanwhile, we should also protect the sensitive areas and promote the construction of green space systems.

From a methodological standpoint, integration is a limited creation based on the holistic and ecological conception. The ideology of the spatial integration put emphasis on the use of the existing forms or constructions. Some of the designers have changed the existing materials into new and useful applications, which happen to go with the same idea of the sustainable design. Zhongshan Shipyard Park built on the site of the abandoned shipyard made use of the rusty machines to create beautiful spaces contrasted with wild grass, which also meets the aesthetic demands of visual ecology.

\section{CONCLUSION}

Modern landscape design has surpassed the traditional definition. Because of the concept of the interactive design, landscape is not only working as the media to overlook the city, but also a media for rebuilding the city. What the city will be in the 21 century? How to create better space experience and high quality environment?

The thinking change should be firstly developed in the designers' consciousness. The interaction in the landscape design is an attitude, a thinking way and an action style. The city landscape should be considered as the process of the living system. A more adaptable and flexible spacial structure and form so as to meet the needs of the future development. Through the study about the interactive design concept and its corresponding design method, urban landscape designers should work obey the scientific design method, integrating all the concepts, and then carry on the quantitative analysis in the media of GIS. This is not the superficial. The key of the interactive landscape design is the interaction between human and nature, not only to improve environment but also to cultivate human nature in the environment.

\section{ACKNOWLEDGMENT}

Humanities and Social Sciences project of the Ministry of Education: Research on the cultural form and ecological planning of the agricultural landscape design in the northeast of China. Project number: 15YJC760015.

Innovative talent training program of the Education Department in Hei Longjiang Province: Research on the ecological urban landscape design based on the Shanshui City concept. Project number: UNPYSCT-2016056.

Arts and Science Program in Hei Longjiang province: Research on the cultural form of the agricultural landscape in the northeast of China. Project number: 2016D032.

Innovative talent training program of Harbin University of Commerce: Research on the Urban Landscape Design Methodology Based on the Shanshui City concept. Project number: 2016QN035. 


\section{REFERENCES}

[1] Пенкин, М., translated by Guangxuan Ren, Arts and ScienceQuestions, Paradox, Research, Culture and Arts Press, Beijing, 1987. pp. 193.

[2] Alan Cooper, Robert Reimann, translated by Jianfeng Zhan, Software conception revolution-The essence of Interactive Design, Electronic Press, Beijing, 2005, pp. 27-35.

[3] Monica G. Turner, Robert H. Gardner, Robert V. O'Neill. Landscape Ecology in theory and practice: Pattern and process, Springer-Verlag New York Inc., New York, 2001.
[4] Affordance and Design, Article from the internet: http://www.hiid.com/?p=2732

[5] Ian McHarg, translated by Jingwei Huang, Design with Nature, Tianjin University Press, Tianjin, 2006.

[6] Kongjian $\mathrm{Yu}$, Lessons learned from the International City Beautification Movement in China, Chinese Landscape Architecture, 2000, pp. 32-35.

[7] Qiang Niu, Application Guide of GIS in the City Planning, China Building Industrial Press, Beijing, 2012.

[8] Donald Arthur Norman, translated by Jinsan Chen, Emotional Design, Electronic Industry Publishing House, 2005. 